\title{
Avaliação da eficiência dos revestimentos condutores para blindagem eletrostática e dissipação da carga estática em um polímero isolante
}

\author{
Alexandros Leonidas Aravanis ${ }^{{ }^{*}}$ \\ Carolina Delwing ${ }^{l}$ \\ Alvaro Meneguzzi ${ }^{1}$
}

\section{Resumo}

Neste trabalho foram processadas tintas base epóxi pigmentadas com polianilina (PAni), que é um polímero intrinsecamente condutor, em diferentes composições. O objetivo foi avaliar a eficiência dos revestimentos condutores em um substrato isolante como o copolímero Acrinonitrila-Butadieno-Estireno (ABS) para blindagem e dissipação da carga estática. Foi realizada uma investigação por análise de infravermelho no revestimento da mistura entre PAni e epóxi, para verificar se houve reações que resultassem em novas ligações químicas. Foi avaliada a distribuição das fibras da PAni e a rugosidade dos revestimentos através de um microscópio eletrônico de varredura e um rugosímetro para analisar a influência dessas características dos filmes na condutividade final das amostras. As medições da condutividade foram feitas por um ensaio em quatro pontas e desse modo foi avaliada a influência da degradação na condutância desses revestimentos. Comparando com indicadores estabelecidos em outros trabalhos se constatou a possibilidade do uso dos revestimentos pigmentados com PAni para blindagem e dissipação da carga estática do material não condutor, que é o copolímero ABS.

Palavras-chave: Polianilina; Carga estática; Polímeros condutores; Revestimentos; Antiestática.

\section{Evaluation of the efficiency of conductor coverings for electrostatical shielding and dissipation of static charge in an insulating polymer}

\begin{abstract}
In this work, epoxy base paints pigmented with polyaniline (PAni), which is an intrinsically conductive polymer, were accomplished in different compositions. The objective was to evaluate an efficient conductive coating on an insulating substrate, such as the Acrylonitrile-Butadiene-Styrene (ABS), for shielding and dissipation of the static charge. An investigation was performed by infrared analysis on the coating of the mixture between PAni and epoxy, to verify if there were reactions that resulted in new chemical bonds. The distribution of the PAni fibers and the roughness of the coatings were evaluated by scanning electron microscopy and rugosimetry to analyze the characteristics of the conductive inks in the samples. Conductivity measurements were carried out at four points and the influence of the degradation on the conductance of these coatings was evaluated. Comparing with the indicators established in other studies, it was possible to use conductive coatings pigmented with PAni for shielding and dissipation of the static load of nonconductive materials.
\end{abstract}

Keywords: Polyaniline; Static charge; Conductive polymer; Coatings; Antistatic.

\section{Introdução}

Atualmente, em ciência e tecnologia dos materiais, associar as propriedades específicas de dois materiais diferentes para o desenvolvimento de um produto é essencial para que este execute o desempenho esperado na finalidade a qual ele é requisitado. Os materiais são classificados como cerâmicos, poliméricos e metálicos. Cada classe de materiais possui determinadas características e propriedades: mecânicas, elétricas, térmicas e ópticas; além de outras características como peso, densidade, resistência a processos de degradação e processabilidade. Os materiais, podendo ser de uma mesma ou de diferentes classes, podem apresentar características adicionais em suas propriedades a partir das misturas com espécies de classes semelhantes ou distintas. Destarte, pode ser adicionada uma característica condutora em um material isolante elétrico [1].

${ }^{1}$ Programa de Pós-graduação em Engenharia de Minas, Metalúrgica e de Materiais, Laboratório de Corrosão, Proteção e Reciclagem de Materiais LACOR, Universidade Federal do Rio Grande do Sul-UFRGS, Porto Alegre, RS, Brasil.

*Autor correspondente: sandrosaravanis044@gmail.com 
Materiais condutores que possuem propriedades antiestáticas despertam interesse nas aplicações tecnológicas por possibilitar blindagem eletrostática no acondicionamento de produtos eletrônicos, e ao mesmo tempo possibilitando a dissipação da carga estática em processos onde a operação requer a utilização de produtos químicos inflamáveis; já que uma centelha oriunda de uma descarga eletrostática entre dois corpos pode gerar explosões [2,3].

Os polímeros são isolantes elétricos que podem acumular carga estática. Eles são compostos por moléculas grandes, que são cadeias de átomos de carbono, das quais vários átomos ou radicais estão lateralmente ligados. Apresentam propriedades não tão rígidas e nem tão fortes como os metais, assim como uma deformação elástica e plástica, por isso não são tão frágeis como alguns materiais cerâmicos. Também apresentam baixa densidade e são considerados materiais leves [4].

Polímeros intrinsecamente condutores (PICs), conhecidos como metais sintéticos, foram desenvolvidos pela primeira vez, por Shirakawa e colaboradores no final de 1970. Devido a esta descoberta, Shirikawa recebeu o prêmio Nobel de Química de 2000 [5]. A polianilina (PAni) é um dos PICs mais estudados por diversas características: alta estabilidade ao meio ambiente, condutividade elétrica relativamente elevada, facilidade de síntese, boa reversibilidade nas reações redox e monômero de baixo custo. $\mathrm{O}$ único problema deste polímero está na processabilidade devido à degradação que ocorre abaixo da temperatura de fusão. A condutividade dos PICs é explanada pelos buracos na cadeia polimérica que dão origem a uma banda de energia que sobrepõe à banda de valência e a de condução, criando uma banda parcialmente preenchida em temperatura ambiente. O mecanismo de condução se dá pela deslocalização dos elétrons $\pi$ que se movem na direção dos buracos portadores de cargas ao longo da cadeia. $\mathrm{O}$ monômero anilina, que dá origem ao polímero polianilina, pode ser processado por uma síntese química, com uso de um ácido protônico funcionalizado (DBSA) em um processo de emulsão em uma única etapa; o resultado da reação é a formação de fibras de PAni ligadas ao DBSA (PAni/DBSA) [6,7].

Com o objetivo de agregar condutividade às características dos polímeros isolantes. As misturas de cargas condutoras em matrizes poliméricas têm demandado diversos estudos no ramo acadêmico. Desse modo, essas misturas oferecem materiais com as características das matrizes poliméricas desejadas e ao mesmo tempo dissipadores de eletricidade estática e com blindagem eletromagnética. Na maioria dos trabalhos propostos, essa mistura se dá no processamento da matriz isolante com a adição das cargas condutoras. Desse modo, acarreta um empecilho no uso da PAni/DBSA como aditivo condutor, já que é um polímero que degrada a baixas temperaturas de fusão $[8,9]$.

Uma proposta diferente desta linha pode ser o uso de um revestimento condutor nas matrizes isolantes sem a necessidade da adição de carga condutora durante a fase de processamento. Assim, a simples mistura da PAni/DBSA em resinas poliméricas criando tintas condutoras, e a aplicação dessas tintas sobre o substrato isolante polimérico, evitaria a degradação devido às altas temperaturas utilizadas durante a fase de processamento da matriz.

Destarte, materiais com propriedades antiestáticas, unidos às características desejadas como as propriedades mecânicas e levezas dos polímeros, podem ser alcançados com um revestimento condutor sobre a superfície de uma matriz polimérica. Os indicadores dos valores satisfatórios de condutividade para os materiais antiestáticos usados para dissipação e blindagem eletrostática estão na faixa de $10^{-5}$ a $10^{-7} \mathrm{~S}^{-\mathrm{cm}^{-1}}[2,7]$.

\section{Materiais e métodos}

\subsection{Materiais}

Neste trabalho foi usado Anilina $\left(\mathrm{C}_{6} \mathrm{H}_{5} \mathrm{NH}_{2}\right)$ da marca Nuclear com 99\% de pureza, ácido dodecil benzeno sulfônico $\left(\mathrm{CH}_{3}\left(\mathrm{CH}_{2}\right)_{11} \mathrm{C}_{6} \mathrm{H}_{4} \mathrm{SO}_{3} \mathrm{H}\right)$-DBSA- da Kintran Chemical Industry Co. Limited e pureza de $96 \%$, Tolueno $\left(\mathrm{C}_{6} \mathrm{H}_{5} \mathrm{CH}_{3}\right)$ da marca Synth com $99,5 \%$ de pureza, persulfato de amônia $\left(\mathrm{NH}_{4}\right)_{2}$-PSA- com $98 \%$ de pureza, resina epóxi monocomponente da marca Araldite e nome comercial GZ-488-N-40-1 e Acrinonitrila-Butadieno-Estireno (ABS) processado na empresa Metalsinos. O ABS foi escolhido por ser um material que se torna rugoso ao ser atacado pelo solvente tolueno, aumentando assim a adesão da resina sobre o substrato.

\subsection{Polimerização da PAni por emulsão}

A Polimerização da PAni/DBSA foi realizada em uma etapa onde foi adicionada $0,06 \mathrm{~mol}$ de anilina em uma emulsão previamente preparada com $200 \mathrm{~mL}$ de água destilada, $0.1 \mathrm{~mol}$ de DBSA e $50 \mathrm{~mL}$ de tolueno, resfriados a $0^{\circ} \mathrm{C}$. Após uma hora de agitação da mistura nestas condições, foi gotejado, lentamente na solução por um intervalo de uma hora, $0.04 \mathrm{~mol}$ do iniciador PSA solubilizado em $50 \mathrm{~mL}$ de água. Em seguida, a reação foi mantida por mais 5 horas [10].

Por fim, $200 \mathrm{~mL}$ de tolueno foram adicionados à solução para finalizar o processo de polimerização. Essa mistura foi deixada em repouso, e após formar uma solução homogênea ocorreu a separação em duas fases: água e solvente. A PAni obtida, dopada com DBSA, permaneceu solubilizada na fase do solvente e o DBSA e o PSA não reagidos ficaram na fase da água como é apresentado na Figura 1.

A PAni/DBSA é separada da água em um funil de separação e transferida para um frasco Erlenmeyer de boca larga.

\subsection{Preparação das tintas}

Foram coletados $10 \mathrm{~mL}$ de PAni/DBSA em um vidro relógio e foi deixado sob ação atmosférica e temperatura ambiente para evaporação do solvente e análise da quantidade de PAni/DBSA. Em 10 mL de PAni/DBSA em solução com solvente havia $1,2469 \mathrm{~g}$ de PAni/DBSA. 


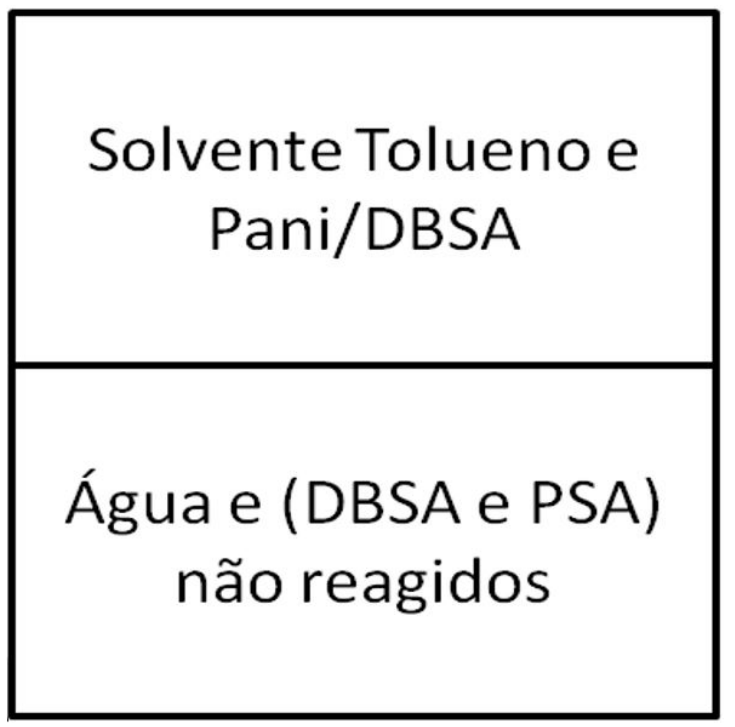

Figura 1. Processo de Solubilização da PAni/DBSA em solvente e Separação da Água.

A resina epóxi também ficou exposta sob ação atmosférica e temperatura ambiente para evaporação do solvente. Para $10 \mathrm{~mL}$ de resina epóxi em solvente foram obtidos 5,80932g de resina epóxi.

Assim, com esses dados, foram produzidas tintas pigmentadas com PAni/DBSA em solução com solvente tolueno com resina epóxi conforme a Tabela 1.

As misturas foram realizadas com a PAni/DBSA em solução com solvente tolueno. Isso, por que a PAni/ DBSA obtida no processo, diluída em tolueno, é mais fácil de homogeneizar na mistura com a resina, por possuir uma viscosidade menor. Como a resina Epóxi apresenta uma viscosidade de $900 \mathrm{mPas}$, isso significa que a medida em que se vai aumentando a quantidade de PAni/DBSA diluída em solvente nas misturas, as viscosidades das tintas serão reduzidas, devido ao adicional de solvente tolueno.

\subsection{Pintura por imersão}

Diferentes amostras de ABS foram pintadas por imersão nas tintas produzidas. A imersão foi de 1 minuto para evitar que o solvente tolueno, presente na PAni/DBSA, ataque o ABS demasiadamente, promovendo a dissolução do polímero e consequentemente a redução de suas propriedades mecânicas. Um baixo ataque do tolueno é esperado, pois gera rugosidade na amostra, aumentando ainda mais a adesão por ancoragem [11].

As amostras de $\mathrm{ABS}$ foram imersas em cada formulação para posterior caracterização.

\subsection{Caracterizações propostas}

A tinta epóxi com 9\% de PAni/DBSA produzida de acordo com a Tabela 1 foi caracterizada por Espectroscopia de Infravermelho por Transformada de Fourier (FTIR), em um equipamento espectrômetro Nexus Tm modelo Thermo
Tabela 1. Composição da Tinta com Resina Epóxi

\begin{tabular}{ccc}
\hline $\begin{array}{c}\text { \%PAni/DBSA na } \\
\text { Resina }\end{array}$ & $\begin{array}{c}\text { PAni/DBSA/ } \\
\text { Solvente }\end{array}$ & Resina Epóxi \\
\hline $9 \%$ & $4,6077 \mathrm{~mL}$ & $10 \mathrm{~mL}$ \\
$19 \%$ & $10,9282 \mathrm{~mL}$ & $10 \mathrm{~mL}$ \\
$30 \%$ & $19,9665 \mathrm{~mL}$ & $10 \mathrm{~mL}$ \\
$40 \%$ & $31,06 \mathrm{~mL}$ & $10 \mathrm{~mL}$ \\
\hline
\end{tabular}

Nicolet, para analisar se a mistura PAni/DBSA e resina epóxi resultou em novas ligações químicas.

As amostras de $\mathrm{ABS}$ que foram pintadas por imersão nas tintas com as composições estabelecidas na Tabela 1, foram submetidas a vários ensaios. Para verificar a dispersão do pigmento PAni/DBSA na resina ao longo do substrato, foi realizada análise em um microscópio eletrônico de varredura da marca ZEISS e modelo MEV EVO MA 10. A espessura do revestimento sobre o substrato foi medida por microscopia óptica em um microscópio Axio LAB. A1 da marca ZEISS. A rugosidade, por um rugosímetro de medição digital modelo RP-200 da marca Instrutherm. A condutividade superficial do substrato, por uma técnica de medição de 4 pontas proposta no artigo de Green e Gunn [12]. Para a influência dos efeitos da degradação, foi proposto um ensaio acelerado em Câmara de Fluorescência Ultravioleta UV. Por fim, o ensaio para verificação das propriedades mecânicas dos revestimentos: impacto e adesão. Os processos serão explanados nos tópicos seguintes referentes aos resultados.

\section{Resultados e discussão}

\subsection{FITR da tinta base epóxi com composição de $9 \%$ de PAni/DBSA}

Análises de Infravermelho foram utilizadas para caracterizar a existência de ligações químicas entre as misturas de resina epóxi e PAni/DBSA. Foram analisados os espectros em escalas diferentes: na Figura 2; entre 3500 a $500 \mathrm{~cm}^{-1}$ e 2000 a $500 \mathrm{~cm}^{-1}$. Os espectros são da PAni/DBSA, da Resina Epóxi, e da Resina Epóxi com PAni/DBSA.

Conforme pode ser visto na Figura 2, as bandas se sobrepuseram, tornando difícil a verificação de novas ligações químicas na mistura da PAni/DBSA com a resina epóxi. Observa-se um espectro muito parecido com a resina epóxi virgem, porém com a presença dos picos característicos da PAni/DBSA.

\subsection{Rugosidade}

Na Figura 3, verifica-se a rugosidade em função da proporção de PAni/DBSA na resina epóxi.

Há um aumento maior na rugosidade na proporção da tinta de 20 a 30\% de PAni/DBSA. A partir da composição de 30\% PAni/DBSA, houve uma estabilização nos valores 
referentes à rugosidade. Além de 30\% houve uma sobreposição das fibras que resultou em um valor mais estável, nos intervalos de proporção de 30 a 40\% PAni/DBSA.

\subsection{Microscopia Eletrônica de Varredura (MEV)}

Na Figura 4 são apresentadas as imagens (a), (b), (c) e (d) com um aumento de 500 vezes na magnitude para os filmes das tintas com a proporção estabelecida na Tabela 1.

$\mathrm{O}$ processo de emulsão resultou na produção de um polímero condutor fibrilar, conforme as imagens da Figura 4. A morfologia superficial da PAni pode apresentar duas estruturas, dependendo do dopante utilizado: granular e fibrilar [13]. No processo de emulsão em uma etapa com o ácido protônico funcionalizado, a PAni/DBSA polimeriza em uma morfologia fibrilar [14].

As imagens mostram um aumento da quantidade e distribuição das fibras na medida em que se aumenta a composição de PAni/DBSA nas resinas.

Nesta análise semiqualitativa foi possível avaliar a distribuição homogênea de fibras na superfície do substrato. Também, as fibras começaram, a partir de certa quantidade de PAni/DBSA na mistura, a precipitar para superfície da solução com a resina, o que justifica o aumento da rugosidade.

\subsection{Microscopia óptica}

Na Figura 5 é apresentada a variação do tamanho médio de espessura dos revestimentos em função do percentual de PAni/DBSA nos substratos imersos nas tintas formuladas conforme Tabela 1, medidos por microscopia óptica.

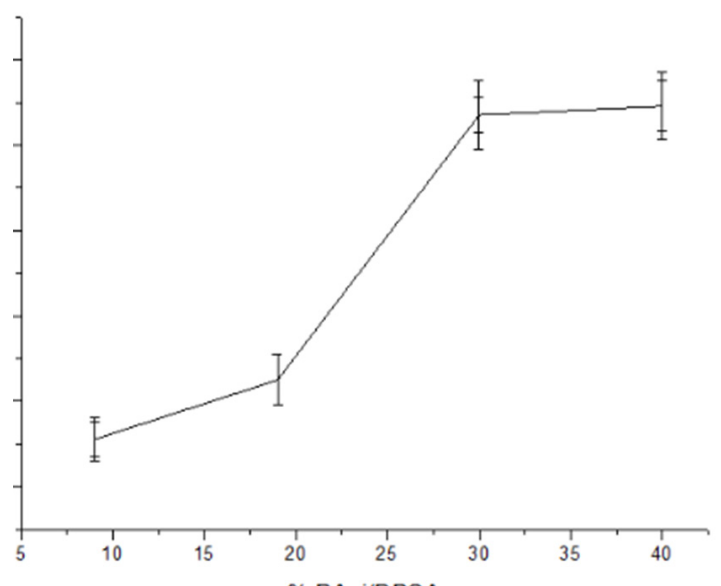

Figura 3. Análise da rugosidade em função do percentual de PAni/ DBSA na resina epóxi.
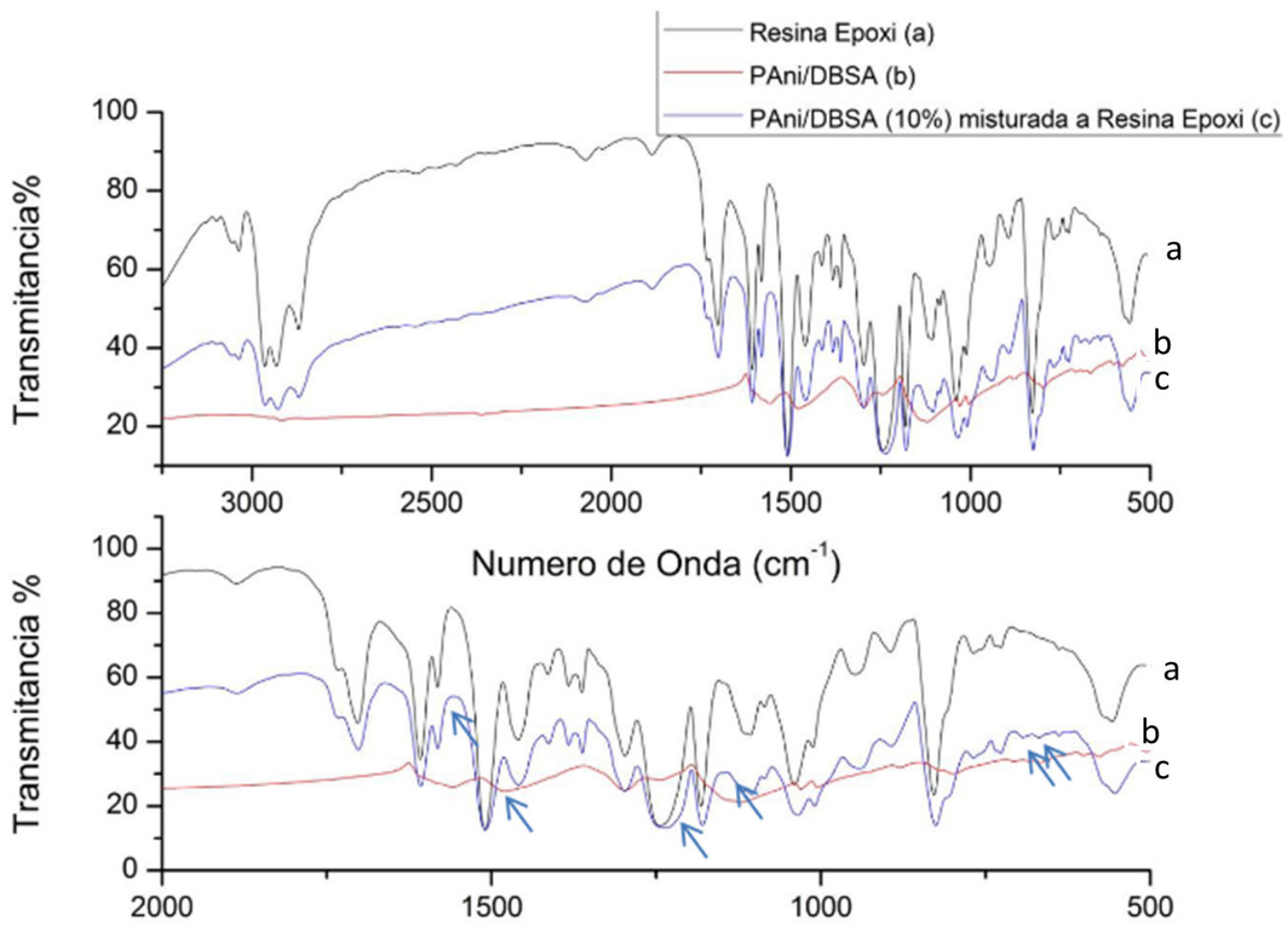

Numero de Onda $\left(\mathrm{cm}^{-1}\right)$

Figura 2. Espectroscopia de Infravermelho: (a) Resina Epóxi; (b) PAni/DBSA; (c) PAni/DBSA misturada a Resina Epóxi. 


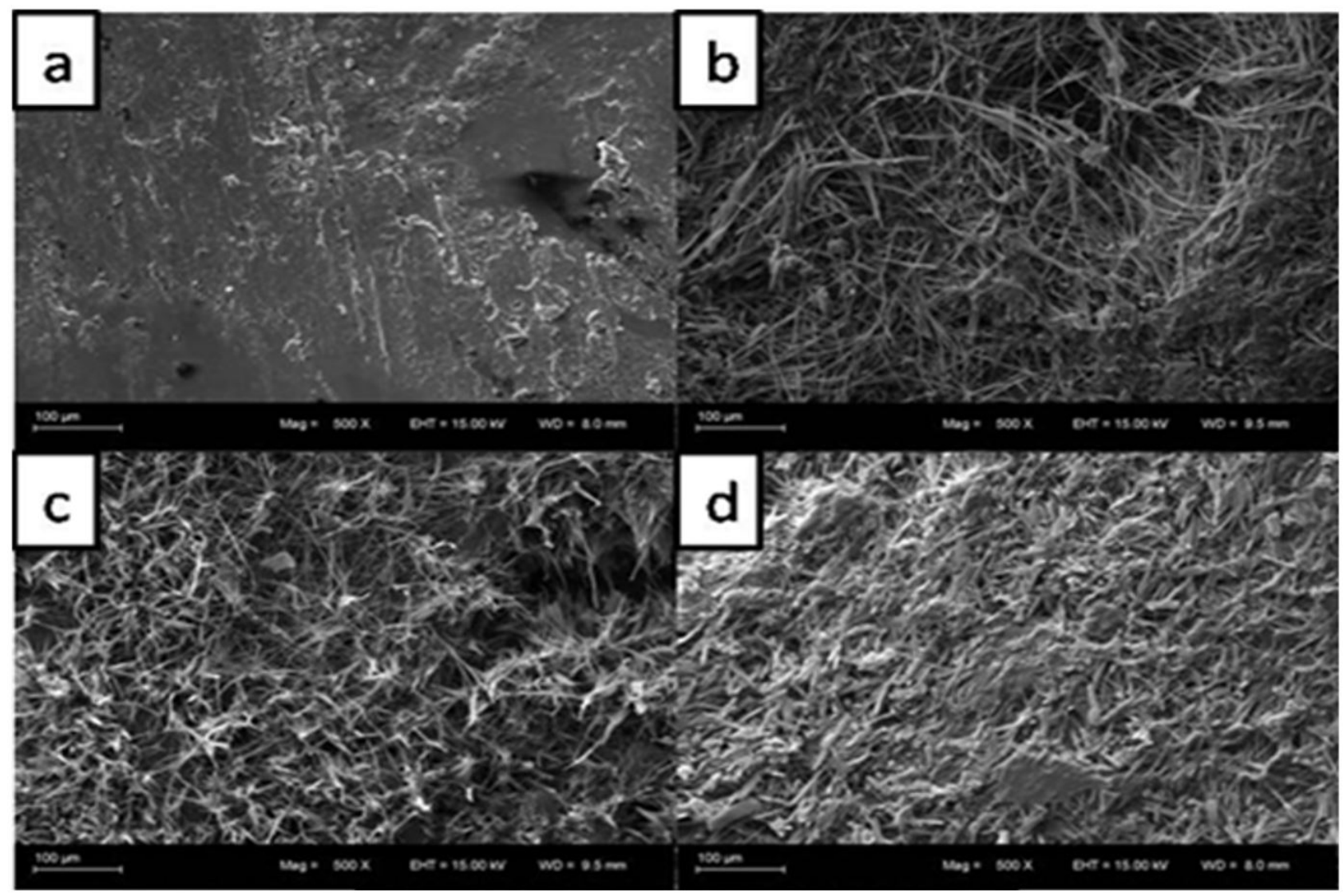

Figura 4. Resina Epóxi com diferentes composições de PAni/DBSA; (a) ABS imerso em tinta base epóxi com 9\% PAni/DBSA; (b) ABS imerso em tinta base epóxi com 19\% PAni/DBSA; (c) ABS imerso em tinta base epóxi com 30\% PAni/DBSA; (d) ABS imerso em tinta base epóxi com 40\%.

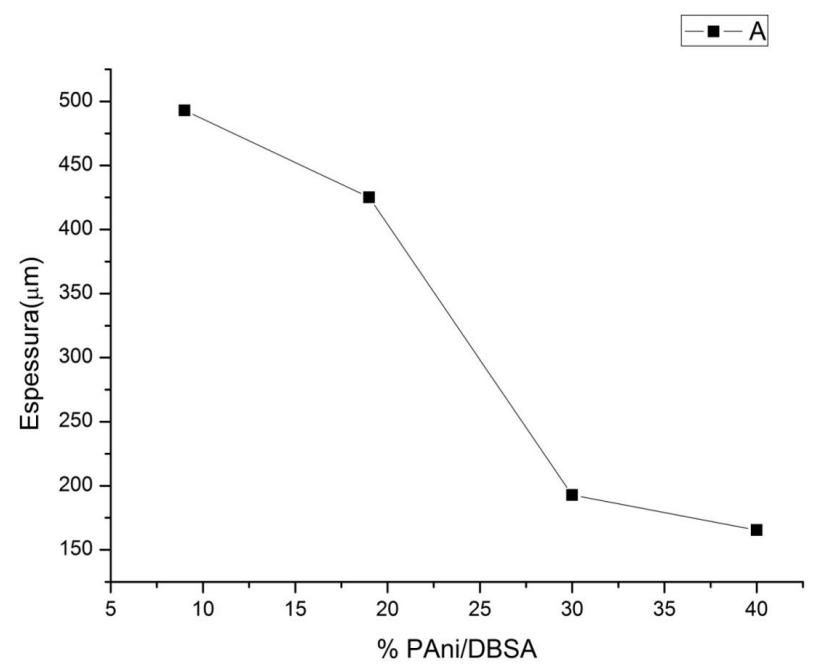

Figura 5. Espessura do revestimento na superfície do substrato em função do percentual de PAni/DBSA na resina

Há uma redução na espessura dos revestimentos obtidos das diferentes formulações, na medida em que se aumenta a quantidade de PAni/DBSA na resina. Para manter a PAni no estado solúvel, o tolueno não é retirado e a maior adição de PAni/DBSA leva a uma maior diluição das tintas obtidas, resultando em diminuição da viscosidade. Este fato se reflete na diminuição da espessura.

\subsection{Análise da condutividade dos revestimentos}

Pela técnica de 4 pontas foram medidas as correntes, em Amperes, e o potencial, em Volts, na superfície dos substratos imersos nas tintas com resina epóxi cujas composições foram apresentadas na Tabela 1 .

Após os ensaios de condutividade, as amostras foram encaminhadas a uma câmara úmida, onde ficaram exposta à radiação UV em três intervalos de tempo de 250 , 500 e 1000 horas, ou seja, as amostras ficaram expostas durante 1000 horas ao ensaio de acordo com a norma ASTM G154-12a [15]. A cada intervalo as amostras eram retiradas da câmara e eram realizadas novas medições da condutividade

Na Tabela 2 são apresentados os dados da Figura 6.

A análise da Tabela 2 deixa claro que todas as amostras apresentaram uma redução na condutividade após mil horas de ensaios, sendo que entre 500 e 1000 horas não foi apresentada uma redução significativa nos valores da condutividade.

A Figura 6 apresenta o $\log$ da condutividade nas amostras não ensaiadas na câmara de fluorescência UV e nas amostras ensaiadas em intervalos de 250, 500 e 1000 horas.

$\mathrm{O}$ efeito da degradação reduziu a condutividade dos revestimentos em todos os intervalos de tempo analisados; sendo mais efetivo nos intervalos de 0 a 500 horas, exceto entre 500 e 1000 horas que não 
Tabela 2. Condutividades no ensaio de degradação acelerado

\begin{tabular}{|c|c|c|c|c|}
\hline \multirow{2}{*}{ Composição } & Sem Ensaio & 250 Horas & 500 horas & 1000 horas \\
\hline & Condutividade (s/cm) & Condutividade (s/cm) & Condutividade (s/cm) & Condutividade (s/cm) \\
\hline $40 \%$ & 2,927808987 & 1,014915236 & 0,041962709 & 0,039421793 \\
\hline $30 \%$ & 0,161180472 & 0,111770259 & 0,011112384 & 0,007225961 \\
\hline $17 \%$ & 0,153504808 & 0,097088635 & 0,004267438 & 0,003093268 \\
\hline $9 \%$ & 0,003370856 & 0,000697895 & 0,000216309 & $8,54 \mathrm{E}-04$ \\
\hline 0 & $3,43 \mathrm{E}-10$ & $3,43 \mathrm{E}-10$ & $3,43 \mathrm{E}-10$ & $3,43 \mathrm{E}-10$ \\
\hline
\end{tabular}

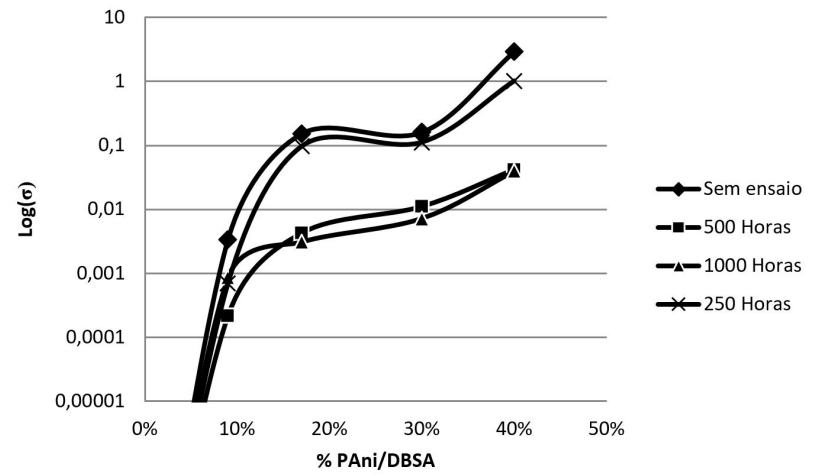

Figura 6. Log da condutividade das amostras para diferentes composições de PAni/DBSA: (a) - $\diamond$ - sem ensaio; (b) -X- ensaio de 250 horas; (c) -- ensaio de 500 horas; (d) $-\Delta$ - Ensaio de 1000 horas.

ocorreu elevada redução da condutividade. Isso ocorre, provavelmente, porque as fibras mais internas na resina não tiveram uma redução nos transportadores de cargas de PAni/DBSA, devido à própria proteção da resina, já as fibras externas degradaram principalmente no intervalo de 0 a 250 horas.

A redução na condutividade da tinta com $40 \%$ PAni/DBSA é mais acentuada entre 250 e 500 horas, diferentemente das outras resinas, isso está associado a menor viscosidade da tinta que oferece a possibilidade de ter fibras mais bem distribuídas internamente no revestimento e assim gerando uma maior condutividade e mais resistência à degradação.

As médias das condutividades apresentadas pelas amostras, mesmo após 1000 horas de ensaio, permanecem sendo suficientes para dispersão da carga estática superficial do polímero ABS isolante, sendo os efeitos degradativos insuficientes para descaracterização das diretrizes da pesquisa que é estabelecer a partir do revestimento condutor a dispersão e blindagem eletrostática do ABS isolante.

\subsection{Avaliação das propriedades mecânicas de adesão}

Foi realizado um ensaio de adesão pull-off em diferentes amostras imersas nas tintas com as composições das Tabelas 1 e são mostradas na Tabela 3.
Na medida em que se aumentou a quantidade PAni/ DBSA na resina, houve uma redução na coesão da tinta. Em maiores quantidades de PAni/DBSA ocorreu falha por coesão no revestimento, que acarretou uma ruptura do próprio revestimento. Os valores de adesão foram satisfatórios de acordo com a norma ASTM D4541-09 [16], principalmente nas tintas com proporção de PAni/DBSA entre $9 \%$ a $30 \%$, chegando próximo a valores de adesão em substratos metálicos. Isso se deve, também, pelo ataque do tolueno no substrato de ABS, criando micro poros, que aumentam a adesão por ancoragem mecânica. Nas tintas com $40 \%$ e 50\% de PAni/ DBSA houve falhas do tipo coesiva, quando a coesão do revestimento falha e não a adesão do revestimento sobre o substrato. Essa falha pode estar relacionada ao aumento da quantidade de tolueno, que reduz a viscosidade das tintas e consequentemente a uniformidade das mesmas; aumentando a rugosidade, apresentando menores espessuras na imersão e uma menor resistência do revestimento. Outro fator que contribui para a falha coesiva é o aumento da quantidade de solvente nas maiores composições de PAni/DBSA, as moléculas tendem a se enrolar como novelos acarretando em um filme com baixa coesão.

\subsection{Avaliação das propriedades mecânicas de impacto}

Para todos os ensaios de impacto houve rompimento da base de polímero $\mathrm{ABS}$, devido à queda do indentador, que consequentemente causou a ruptura do revestimento. Pode-se concluir que os filmes com a resina epóxi tiveram uma resistência ao impacto superior ao próprio substrato de $\mathrm{ABS}$, portanto superior ao mínimo requerido para aplicação desta tinta sobre este substrato.

\section{Conclusões}

Não foi possível averiguar se houve ligação química entre a PAni/DBSA e a resina devido à sobreposição das bandas de energia na análise de FTIR.

Com aumento da quantidade de PAni/DBSA, aumentou a quantidade de fibras de PAni/DBSA sobre o substrato. Consequentemente reduziu a espessura das tintas e aumentou a rugosidade. Os resultados mostraram que a condutividade 
Tabela 3. Análise da Adesão para as os filmes obtidos com as tintas produzidas de acordo com a Tabela 1

\begin{tabular}{|c|c|c|}
\hline Proporção & Tensão de Ruptura & \multirow{2}{*}{ Tipo de Falha } \\
\hline PAni/DBSA (\%) & (MPa) & \\
\hline 9 & 1,26 & Falhou a cola que liga a dolly ao substrato \\
\hline 17 & 1,10 & Falha adesiva perfeita \\
\hline 30 & 1,04 & Falha adesiva perfeita \\
\hline 40 & 0,85 & Falha coesiva na tinta \\
\hline 50 & 0,63 & Falha $100 \%$ coesiva \\
\hline
\end{tabular}

está relacionada principalmente com a quantidade de PAni/ DBSA na mistura polimérica do aditivo condutor e não com a espessura e rugosidade do filme sobre o substrato. O Efeito de degradação na condutividade foi maior com proporção de $40 \%$ PAni/DBSA nos intervalos de tempo de 250 a 500 horas em exposição a câmara de Fluorescência Ultravioleta. Nas outras composições após 250 horas foi registrado uma redução significativa da condutividade. A partir de $30 \%$ as amostras apresentaram um aumento significativo de condutividade. No ensaio de 1000 horas houve uma redução na condutividade significativa em todas as composições devido ao menor número de portadores de cargas e menor contato entre as fibras de PAni/DBSA.
Nos ensaios de adesão, as tintas apresentaram uma ruptura mais coesiva à medida em que se aumentou a quantidade de PAni/DBSA na mistura. Já em impacto, todas as tintas apresentaram bons resultados, sendo necessária a ruptura do substrato polimérico para causar danos na tinta.

Os resultados foram satisfatórios em relação à condutividade e propriedades mecânicas dos filmes sobre o substrato, apresentando condutividades maiores que os indicadores esperados na faixa de $10^{-5} \mathrm{a} 10^{-7} \mathrm{~S} . \mathrm{cm}^{-1}$ alcançados em outros trabalhos acadêmicos onde se enxertou material condutor na matriz polimérica durante a etapa de processamento. Por isso, o revestimento é uma alternativa que pode ser capaz de blindar estaticamente e dispersar a energia estática do polímero ABS.

\section{Referências}

1 Callister WD. Materials science and engineering: an Introduction. 7th ed. New York: LTC; 2007.

2 Rosa BS. Estrutura e propriedades de misturas poliuretano termoplástico com aditivos condutores [dissertação]. Florianópolis: Universidade Federal de Santa Catarina; 2014.

3 Mattos UAO. Higiene e segurança do trabalho. 1. ed. Rio de Janeiro: Elsevier e ABEPRO; 2011.

4 Mano EB, Mendes LC. Introdução a polímeros. 2. ed. São Paulo: LTC; 2007.

5 Valdes LB. Resistivity measurements on germanium for transistors. Proceedings of the IRE. 1954;42(2):420-427.

6 Meneguzzi A. Eletrosíntese de filmes de polímeros a partir de naftilaminas substituidas sobre metais oxidáveis e síntese química: aplicação na proteção contra a corrosão [tese]. Porto Alegre: Universidade Federal do Rio Grande do Sul; 2000.

7 Mitzakoff S. Blendas de polianilina e plásticos de engenharia [dissertação]. Campinas: Universidade Estadual de Campinas; 1997.

8 Martins CR, De Paoli MA. Antistatic thermoplastic blend of polyaniline and polystyrene prepared in a double-screw extruder. European Polymer Journal. 2005;41(12):2867-2873.

9 Ramôa SDA. Preparação e caracterização de compósitos de poliuretano termoplástico com negro de fumo condutor e nanotubos de carbono [dissertação]. Florianópolis: Universidade Federal de Santa Catarina; 2011.

10 Österholm JE, Cao Y, Klavetter F, Smith P. Emulsion polymerization of aniline. Polymer. 1993;55(2):1034-1039.

11 Selin N. Ánalise da superfície de polímeros pós-tratamento corona [dissertação]. Florianópolis: Universidade Federal de Santa Catarina; 2012.

12 Green MA, Gunn M. Four point probe hall effect and resistivity measurements upon semiconductors. Solid-State Electronics. 1972;15(2):577-585.

13 Luiz Henrique M. Polianilinas: síntese, estrutura e propriedades. Quimica Nova. 1996;19(4):388-399.

14 Zhang Q, Jin H, Wang X, Jing X. Morphology of conductive blend fibers of polyaniline and polyamida-11. Synthetic Metals. 2001;123(3):481-485.

15 American Society for Testing and Materials. ASTM G154-12a. West Conshohocken: ASTM.

16 American Society for Testing and Materials. ASTM D4541-09. West Conshohocken: ASTM.

Recebido em: 24 Set. 2019

Aceito em: 15 Maio 2020 